\title{
Multiplicative Renormalizability of Operators defining Quasiparton Distributions
}

\author{
Zheng-Yang Li, ${ }^{1, *}$ Yan-Qing Ma, ${ }^{1,2,3, \dagger}$ and Jian-Wei Qiu ${ }^{4, \$}$ \\ ${ }^{1}$ School of Physics and State Key Laboratory of Nuclear Physics and Technology, Peking University, Beijing 100871, China \\ ${ }^{2}$ Center for High Energy physics, Peking University, Beijing 100871, China \\ ${ }^{3}$ Collaborative Innovation Center of Quantum Matter, Beijing 100871, China \\ ${ }^{4}$ Theory Center, Jefferson Lab, 12000 Jefferson Avenue, Newport News, Virginia 23606, USA
}

(Received 17 September 2018; published 15 February 2019)

\begin{abstract}
Extracting parton distribution functions (PDFs) from lattice QCD calculation of quasi-PDFs has been actively pursued in recent years. We extend our proof of the multiplicative renormalizability of the quasiquark operators of Ishikawa et al. [Phys Rev. D 96, 094019 (2017)] to quasigluon operators, and demonstrated that quasigluon operators could be multiplicatively renormalized to all orders in perturbation theory, without mixing with other operators. We find that using a gauge-invariant UV regulator is essential for achieving this proof. With the multiplicative renormalizability of both quasiquark and quasigluon operators, and QCD collinear factorization of hadronic matrix elements of there operators into PDFs, extracting PDFs from lattice QCD calculated hadronic matrix elements of quasiparton operators could have a solid theoretical foundation.
\end{abstract}

DOI: 10.1103/PhysRevLett.122.062002

Introduction.-The parton distribution functions (PDFs) encode important nonperturbative information of strong interactions. Based on QCD factorization [1], PDFs have been successfully extracted from high-energy scattering data with a good precision [2]. However, from both theoretical and practical points of view, extracting PDFs from firstprinciples lattice QCD (LQCD) calculations must be done for testing the nonperturbative sector of QCD, as well as being needed for studying partonic structure of hadrons that could be difficult to do scattering experiments with.

Calculating PDFs in Euclidean-space LQCD directly, if not impossible, is difficult due to the time dependence of the operators defining PDFs [2]. A novel approach was suggested by $\mathrm{Ji}$ [3], who introduced a set of LQCDcalculable quasi-PDFs and argued that the quasi-PDFs of hadron momentum $P_{z}$ become corresponding PDFs when $P_{z}$ is boosted to infinity. A number of other approaches to extract PDFs from LQCD calculations were also proposed [4-8]. In Refs. [9,10], two of us proposed a QCD factorization based general approach to calculate PDFs in LQCD indirectly. Similar to the extraction of PDFs from experimental data of factorizable and measurable hadronic cross sections, we proposed to extract PDFs by the global analysis of data generated by LQCD calculations of good "lattice cross sections" (LCSs), which are defined as hadronic matrix elements that satisfy (1) being calculable

Published by the American Physical Society under the terms of the Creative Commons Attribution 4.0 International license. Further distribution of this work must maintain attribution to the author(s) and the published article's title, journal citation, and DOI. Funded by SCOAP ${ }^{3}$. in Euclidean-space LQCD, (2) being renormalizable for ultraviolet (UV) divergences to ensure a reliable continue limit, and (3) being factorizable to PDFs with infrared-safe matching coefficients. It is the (3) factorization that relates the desired PDFs to the LQCD calculable LCSs.

To extract the rich, precise, and flavor separated information on PDFs, it is necessary to find as many good LCSs as possible, since different flavor PDFs are likely to contribute to the same LQCD calculated LCSs. For constructing good LCSs, we studied two types of operators in terms of (1) the correlation of two gauge-dependent field operators with proper gauge links, which we call quasiparton operators since they cover all operators defining quasi-PDFs and more, and (2) the correlation of two gaugeinvariant currents. In our approach, the LQCD calculation of each of these good LCSs in coordinate space provides the needed information to constrain the PDFs, similar to the role of measuring various experimental cross sections to constrain the PDFs. For a comparison, in Ji's proposal [3], one focuses on reproducing PDFs by corresponding quasiPDFs calculated in LQCD at a large enough $P_{z}$.

For LQCD calculations, it is relatively less expensive to calculate the type (1) operators comparing with type (2) operators, while the renormalization of the type (2) operators is much more simpler than that of the type (1) operators. Renormalization of the type (2) operators is almost trivial, for which one only needs to renormalize the gaugeinvariant local currents, which is well known. On the other hand, the renormalization of the type (1) operators is nontrivial due to the nonlocality of corresponding operators and potential power divergences. QCD factorization of both types of operators have been studied in Ref. [10], in 
which we found that multiplicative renormalizability of these operators is a necessary condition for the collinear factorization to be valid.

A lot of effort has been devoted to explore the UV structure of the type (1) quasiquark operators [11-18]. All-order multiplicative renormalizability of quasiquark operators has been proven using two different methods: one relies on the auxiliary field technique $[19,20]$, and the other is based on diagrammatic expansion [21]. These proofs provided a firm theoretical basis for extracting the combination of quark distributions, such as $u(x)-d(x)$, that are not sensitive to gluons from LQCD calculated hadronic matrix elements of quasiquark operators [22-38].

The UV structure of quasigluon operators could be much more complicated, as we will explain. We define general bare quasigluon operators as

$$
\mathcal{O}_{b g}^{\mu \nu \rho \sigma}(\xi)=F^{\mu \nu}(\xi) \Phi^{(a)}(\{\xi, 0\}) F^{\rho \sigma}(0),
$$

where $\Phi^{(a)}(\xi, 0)=\mathcal{P} e^{-i g_{s} \int_{0}^{1} \xi \cdot A^{(a)}(r \xi) d r}$ is a path ordered gauge link in adjoint representation. To be definite, we assume $\xi_{\mu}$ along the $z$ direction and introduce a unit vector $n^{\mu}=(0,0,0,1)$, defining $v \cdot n \equiv v_{z}$ for any vector $v_{\mu}$. Due to the dimensional derivative operator in $F^{\mu \nu}$, superficial power counting tells us that the vertex between gluon field strength and gauge link could be linearly UV divergent. By using a cutoff regularization, one-loop calculation in Refs. [39,40] indeed shows uncanceled linear divergences for this vertex, which would make the multiplicative renormalization of quasigluon operators almost impossible.

Another complication comes from operator mixing via UV renormalization. In principle, the general quasigluon operator in Eq. (1) could have 36 independent operators after taking into account the antisymmetry of gluon field strength, and all of them could be mixed under renormalization. In literature, quasigluon PDFs are constructed from linear combinations of $\mathcal{O}_{b g}^{\mu \nu \rho \sigma}[3,40,41]$, whose renormalization is nontrivial due to the mixing of these operators.

In this Letter, we first perform an explicit one-loop calculation of quasigluon operators of an asymptotic gluon of momentum $p$, defined as $\left\langle g(p)\left|\mathcal{O}_{b g}^{\mu \nu \rho \sigma}(\xi)\right| g(p)\right\rangle$. We use dimensional regularization (DR) to regularize both logarithmic and linear UV divergences, which respectively appear as poles around $d=4$ and $d=4-1 / n$ at $n$-loop order. We find that linear UV divergences of one-loop correction to the gluon-gauge-link vertex are canceled under DR, which makes the multiplicative renormalizability of quasigluon operators a possibility. We then explore all possible UV divergent topologies of higher order diagrams. Using gauge invariance, we find that all linear UV divergences from the gluon-gauge-link vertex are canceled to all orders in perturbation theory. Then, we find that all of the 36 independent quasigluon operators can be multipliticatively renormalized without mixing with any other operators. Combining with our proof for quasiquark operators in Ref. [21], our Letter completes the proof of multipliticative renormalization of the quasiparton operators.

$U V$ divergences at one loop.-We present the relevant one-loop Feynman diagrams for quasigluon operators of an asymptotic gluon of momentum $p$ in Fig. 1, where the bubble in the diagram of Fig. 1(e) includes all one-loop self-energy diagrams of the active gluon. For the complete one-loop contribution, additional Feynman diagrams are needed. Some of them are mirror diagrams of Figs. 1(b)-1(e) and 1(g), while the rest can be obtained by replacing external momentum $p$ to $-p$ in all these Feynman amplitudes. For the following one-loop calculation, we take the linearly combined quasigluon operator in Ref. [3] as an example, but our conclusion is true for any of the 36 independent operators.

We choose Feynman gauge, and assume $\xi_{z}$ to be positive for definiteness. Figure 1(a) gives

$$
\begin{aligned}
M_{1 a} & =\frac{g_{s}^{2} \mu_{r}^{4-d} C_{A}}{i} e^{-i p_{z} \xi_{z}} \int_{0}^{\xi_{z}} d r_{1} \int_{r_{1}}^{\xi_{z}} d r_{2} \frac{d^{d} l}{(2 \pi)^{d}} \frac{e^{i l_{z}\left(r_{2}-r_{1}\right)}}{l^{2}} \\
& =\frac{\mathrm{UV}}{\pi} \frac{\alpha_{s} C_{A}}{\pi} e^{-i p_{z} \xi_{z}}\left(-\frac{\pi \mu_{r} \xi_{z}}{3-d}+\frac{1}{4-d}\right),
\end{aligned}
$$

where $\mu_{r}$ is a renormalization scale to compensate the mass dimension in DR.

To understand where in this one-loop phase space the UV divergences in Eq. (2) come from, it is instructive to distinguish $l_{z}$-the $z$ component of the loop momentum $l$ from $\bar{l}_{\mu}$-the other components of $l$, as $l_{\mu}=\bar{l}_{\mu}-l_{z} n_{\mu}$ with $l^{2}=\bar{l}^{2}-l_{z}^{2}$ [21]. If $\bar{l}^{2}$ is constrained in a finite region in Eq. (2), integrating $l_{z}, r_{1}$ and $r_{2}$ cannot generate any UV divergence. Furthermore, there is no UV divergence if we do not include the region where $\left|r_{2}-r_{1}\right|$ is very small,

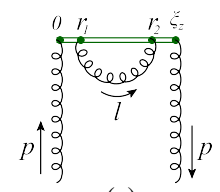

(a)

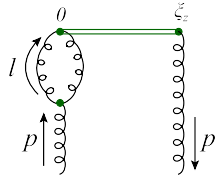

(d)

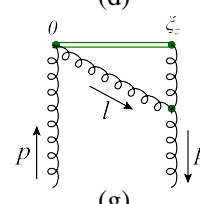

(g)

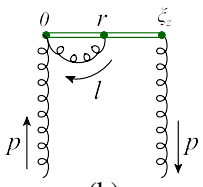

(b)

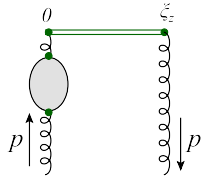

(e)

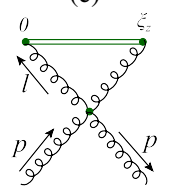

(h)

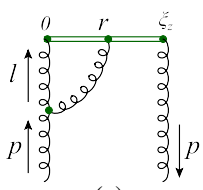

(c)

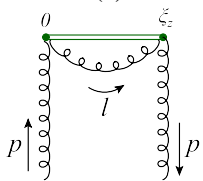

(f)

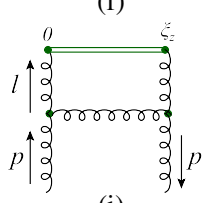

(i)
FIG. 1. Some typical Feynman diagrams for quasigluon PDFs of an asymptotic gluon of momentum $p$ at one-loop order. 
which can be demonstrated by introducing the following decomposition,

$$
\frac{1}{l^{2}}=\frac{1}{\bar{l}^{2}}+\frac{l_{z}^{2}}{l^{2} \bar{l}^{2}},
$$

where the first term is free of $l_{z}$, and thus the integration of $l_{z}$ gives $\delta\left(r_{2}-r_{1}\right)$, while the second term is UV finite under the integration of $\bar{l}$. That is, the UV divergence in Eq. (2) can only come from the region of phase space where $\bar{l}$ are in UV region while $\left|r_{2}-r_{1}\right|$ is very small. Therefore, we conclude that, with DR, all UV divergences of Fig. 1(a) come from a region localized in spacetime.

By decomposing both $1 / l^{2}$ and $\left[1 /(p-l)^{2}\right]$ using Eq. (3), we obtain many terms for Figs. 1(b) and 1(c) and found that these terms are either free of $l_{z}$ in denominator, which result in $\delta(r)$ or its derivatives, or UV finite under the integration of $\bar{l}$. Thus the UV divergences of these two diagrams are also localized in spacetime,

$$
\begin{gathered}
M_{1 b} \stackrel{\mathrm{UV}}{=} \frac{\alpha_{s} C_{A}}{\pi} e^{-i p_{z} \xi_{z}}\left(\frac{-i}{p_{z} \xi_{z}} \frac{\pi \mu_{r} \xi_{z}}{3-d}\right), \\
M_{1 c} \stackrel{\mathrm{UV}}{=} \frac{\alpha_{s} C_{A}}{\pi} e^{-i p_{z} \xi_{z}}\left(\frac{i}{p_{z} \xi_{z}} \frac{\pi \mu_{r} \xi_{z}}{3-d}+\frac{3}{4} \frac{1}{4-d}\right),
\end{gathered}
$$

where Fig. 1(b) has only linear UV divergence, while 1(c) has both linear and logarithmic UV divergence.

Figures 1(d) and 1(e) have only logarithmic UV divergences, which come from the region where all components of $l^{\mu}$ go to infinity, and, thus, is localized in spacetime. All other diagrams in Fig. 1 are free of UV divergence, simply because the loop cannot be localized in spacetime due to finite $\xi_{z}$, same as the argument for quasiquark operators [21]. In summary, we conclude that the UV divergences of quasigluon operators at one loop can only be emerged from a region localized in coordinate spacetime. For a comparison, UV divergences of operators defining PDFs come from a region nonlocal along the "-" light-cone direction [21].

The linear UV divergence in Eq. (2) from Fig. 1(a) is harmless $[21,42,43]$. However, the presence of linear UV divergence in Eqs. (4) and (5) from Figs. 1(b) and 1(c), respectively, could challenge the multiplicative renormalizability. Fortunately, we find that with DR, the linear UV divergences from these two diagrams are canceled. On the contrary, the linear divergences from Figs. 1(b) and 1(c) do not cancel if one uses a cutoff regularization that breaks the gauge symmetry $[39,40]$. This implies that gauge invariance plays an important role to remove the linear UV divergences that may challenge the multiplicative renormalizability. In the following, we will use gauge invariance to show that all linear divergences, except that from the self-energy of gauge links, are canceled by summing over all contributions, and quasigluon operators could be multiplicatively renormalized.

$U V$ divergences at high orders.-From the one-loop diagrams in Fig. 1, we can generate all high order loop diagrams by adding gluons, quark-antiquark pairs, or ghost-antighost pairs to them. Because of the isolation of the $z$ component in the definition of quasiparton operators, both three-dimensional (3D) and 4D integration of loop momentum $\bar{l}$ and $l$ could lead to UV divergence. In Ref. [21], we introduced the change of divergence index $\Delta \omega_{3}$ and $\Delta \omega_{4}$ for the $3 \mathrm{D}$ and $4 \mathrm{D}$ integration of loop momenta of higher order diagrams, respectively, and showed that it is sufficient, although it is not necessary, that quasiparton operators are renormalizable if $\Delta \omega_{3} \leq 0$ and $\Delta \omega_{4} \leq 0$ are satisfied for all corresponding higher order diagrams. Based on the power counting rules derived in Ref. [21], we find that the only case that may increase superficial UV divergence of quasigluon operators at high orders is when we add a gluon with both ends of it attached to the gauge link, where the 3D integration gets $\Delta \omega_{3}=1$. By applying the decomposition in Eq. (3) to the added gluon's momentum, it is straightforward to show that dimensional regularized UV divergences at any loop level are localized in spacetime, in the same way as the quasiquark operators shown in Ref. [21]. As a result, we find that $\Delta \omega_{3}$ is effectively irrelevant for studying UV divergences. Because $\Delta \omega_{4} \leq 0$ for all cases, there are only finite topologies of high order diagrams in Fig. 2 that have UV divergences.

The blobs with topologies in Figs. 2(a) and 2(c) denote one-particle-irreducible diagrams, and they both have linear superficial UV divergences. Because of the potential linear UV divergences, diagrams with one more gluon attached to the blobs can generate logarithmic UV divergences. Another possibility to produce logarithmic divergences is when a gluon is attached to the gauge link outside of, but very close to, the blobs, as shown in Figs. 2(b) and 2(d), with the attachment denoted by a triangle. The blobs of (a)

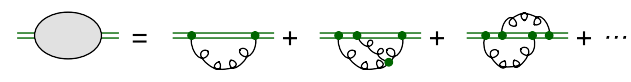

(b)

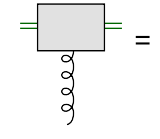

(c)

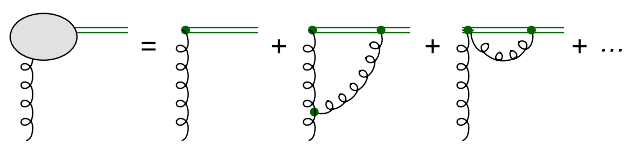

(d)

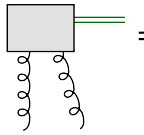

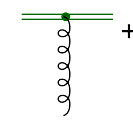
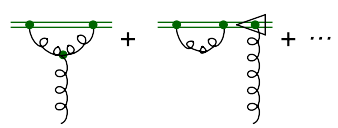

$\overline{2(10)}$
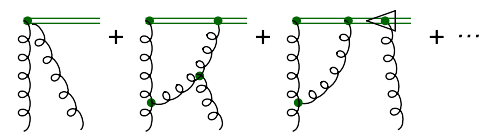

FIG. 2. Four topologies of diagrams that could give UV divergences to the quasigluon operators. 
topologies in Figs. 2(b) and 2(d) include both kinds of logarithmic divergent diagrams mentioned here.

The topologies in Figs. 2(a) and 2(b) are the same as that for quasiquark operators, and their divergences can be renormalized similarly. Linear divergences from the diagrams of the topology in Fig. 2(a) can be removed by an overall factor as the mass renormalization of a test particle moving along the gauge link [42], and its logarithmic divergences caused by end points of the gauge link can be removed by multiplying $Z_{w g}^{-1 / 2}$ - the "wave function" renormalization of the test particle [43]. The diagrams of the topology in Fig. 2(b) has only logarithmic UV divergence, which can be taken care of by QCD renormalization [43].

The UV divergences from diagrams of topologies in Figs. 2(c) and 2(d) are different from that of quasiquark operators, and are studied in next two sections, respectively.

Renormalization of gluon-gauge-link vertex.-For the definiteness of the following discussion, we assume that the gauge link in diagrams of the topology of Fig. 2(c) starts at an arbitrary coordinate $\xi_{1 z}$ with the operator $F^{\mu \nu}\left(\xi_{1 z}\right)$, and ends at another arbitrary coordinate $\xi_{2 z}$ with no additional operators. With the "bare" coupling constant $g_{s}$, and "bare" field operators for the gluons, the Faddeev-Popov ghost and the antighost given by the symbols $A, c$, and $\bar{c}$, respectively, a generalized Ward identity of the non-Abelian field relevant to this topology can be derived [44],

$$
\begin{aligned}
& \left\langle-i \partial_{\lambda}^{y} A_{d}^{\lambda}(y)\left[\Phi\left(\left\{\xi_{2 z}, \xi_{1 z}\right\}\right)\right]_{a b} F_{b}^{\mu \nu}\left(\xi_{1 z}\right)\right\rangle \\
& \quad=\left\langle g_{s} \bar{c}_{d}(y) c_{e}\left(\xi_{2 z}\right)\left[t_{e} \Phi\left(\left\{\xi_{2 z}, \xi_{1 z}\right\}\right)\right]_{a b} F_{b}^{\mu \nu}\left(\xi_{1 z}\right)\right\rangle,
\end{aligned}
$$

where the $t$ represents $\mathrm{SU}(3)$ generators of the adjoint representation. A pictorial representation of Eq. (6) is given in Fig. 3, where "1PR" denotes one-particle-reducible diagrams. The topology of the left-hand side of Fig. 3 is the same as that of Fig. 2(c), but is contracted with external gluon momentum and expressed in coordinate space. The topology of the first term on the right-hand side of Fig. 3 is nonlocal in spacetime, and thus has no UV divergence. Furthermore, after the renormalization of QCD Lagrangian and gauge-link-related topologies of Figs. 2(a) and 2(b), UV divergences of 1PR diagrams will be canceled. That is, the generalized Ward identity in Eq. (6) ensures that the topology of Fig. 2(c) is free of UV divergence if it is contracted by external gluon momentum.

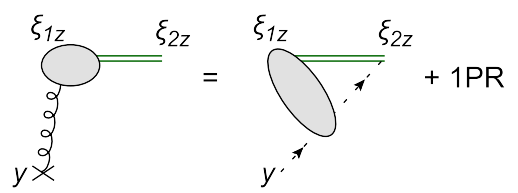

FIG. 3. Pictorial representation of the generalized Ward identity in Eq. (6). The dashed line represents the ghost field.
We represent the gluon-gauge-link vertex of topology in Fig. 2(c) as $\Gamma^{\lambda \mu \nu}(p, n)$, where $p$ and $\lambda$ are the momentum and Lorentz index of the external gluon, respectively. Lorentz symmetry combined with antisymmetry between $\mu$ and $\nu$ enable us to do the general decomposition $\Gamma^{\lambda \mu \nu}(p, n)=\sum_{i=1}^{4} c_{i} \Pi_{i}^{\lambda \mu \nu}$ with

$$
\begin{aligned}
& \Pi_{1}^{\lambda \mu \nu}=g^{\mu \lambda} p^{\nu}-g^{\nu \lambda} p^{\mu}, \quad \Pi_{2}^{\lambda \mu \nu}=\left(p^{\mu} n^{\nu}-p^{\nu} n^{\mu}\right) n^{\lambda}, \\
& \Pi_{3}^{\lambda \mu \nu}=\left(p^{\mu} n^{\nu}-p^{\nu} n^{\mu}\right) p^{\lambda}, \quad \Pi_{4}^{\lambda \mu \nu}=g^{\mu \lambda} n^{\nu}-g^{\nu \lambda} n^{\mu} .
\end{aligned}
$$

Since $p_{\lambda} \Gamma^{\lambda \mu \nu}=0$ for the UV divergent terms as discussed above, we obtain $c_{2} p \cdot n+c_{3} p^{2}+c_{4}=0$, and consequently,

$$
\begin{aligned}
\Gamma^{\lambda \mu \nu}(p, n)= & c_{1} \Pi_{1}^{\lambda \mu \nu}+c_{2}\left(\Pi_{2}^{\lambda \mu \nu}-p \cdot n \Pi_{4}^{\lambda \mu \nu}\right) \\
& +c_{3}\left(\Pi_{3}^{\lambda \mu \nu}-p^{2} \Pi_{4}^{\lambda \mu \nu}\right) .
\end{aligned}
$$

Since $c_{1}$ and $c_{2}$ have mass dimension 0 , locality of UV divergences ensures that they can be at most logarithmic divergent, while $c_{3}$ is UV finite due to its mass dimension at -1 . The only potential linearly UV divergent coefficient $c_{4}$ is removed by gauge invariance. We have therefore demonstrated that the cancellation of linear UV divergences of Figs. 1(b) and 1(c) at one-loop order can be generalized to all orders, which makes the multiplicative renormalizability of quasigluon operators a possibility.

At the lowest order in $\alpha_{s}$, we have $\Gamma^{\lambda \mu \nu}(p, n) \propto \Pi_{1}^{\lambda \mu \nu}$. If we want $\Gamma^{\lambda \mu \nu}(p, n)$ not to mix with other operators under renormalization, we need its UV divergence to be proportional to $\Pi_{1}^{\lambda \mu \nu}$ to all orders. Fortunately, it is always true. For the case with $\mu$ (or $\nu$ ) along the $z$ direction or the case with both $\mu$ and $\nu$ not along the $z$ direction, the coefficients of $c_{2}$ are proportional to $\Pi_{1}^{\lambda \mu \nu}$ or equal to zero, respectively. Therefore, the components of $\Gamma^{\lambda \mu \nu}(p, n)$ do not mix with each other at all, although two different renormalization constants are needed for the two different choices.

In summary, if we choose either $F^{z \bar{\nu}}$ or $F^{\bar{\mu} \bar{\nu}}$ for a gluongauge-link vertex, $\Gamma^{\lambda \mu \nu}$, we can remove the UV divergences of the vertex by multiplying a corresponding renormalization factor $Z_{v g 1}^{-1 / 2}$ or $Z_{v g 2}^{-1 / 2}$, respectively.

Renormalization of gluon-gluon-gauge-link vertex.Finally, we examine the renormalization of the gluongluon-gauge-link vertex of topology in Fig. 2(d). Similar to Eq. (6), we construct the following Ward identity for the "bare" fields and operators,

$$
\begin{aligned}
& \left\langle\partial_{\lambda}^{x} A_{d}^{\lambda}(x) \partial_{\rho}^{y} A_{e}^{\rho}(y)\left[\Phi\left(\left\{\xi_{2 z}, \xi_{1 z}\right\}\right)\right]_{a b} F_{b}^{\mu \nu}\left(\xi_{1 z}\right)\right\rangle \\
& \quad+i \delta^{(d)}(x-y) \delta_{d e}\left\langle\left[\Phi\left(\left\{\xi_{2 z}, \xi_{1 z}\right\}\right)\right]_{a b} F_{b}^{\mu \nu}\left(\xi_{1 z}\right)\right\rangle \\
& =g_{s}\left\langle f^{a f g} \bar{c}_{e}(y) c_{f}\left(\xi_{2 z}\right) \partial_{\lambda}^{x} A_{d}^{\lambda}(x)\left[\Phi\left(\left\{\xi_{2 z}, \xi_{1 z}\right\}\right)\right]_{g b} F_{b}^{\mu \nu}\left(\xi_{1 z}\right)\right\rangle .
\end{aligned}
$$




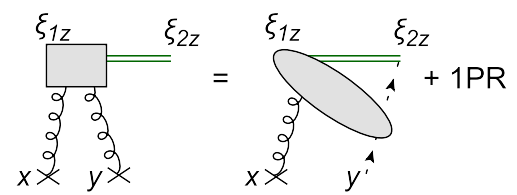

FIG. 4. Pictorial interpretation of the generalized Ward identity in Eq. (9) with dashed line represents the ghost field.

A pictorial interpretation of Eq. (9) is given in Fig. 4. Similar to Fig. 3, the topology of the left-hand side of Fig. 4 is the same as that of the diagram in Fig. 2(d), except the external gluons of the diagrams are contracted with their respective momenta and expressed in coordinate space. The right-hand side of the equation in Fig. 4 is UV finite after all previously discussed renormalizations performed, including QCD Lagrangian, gauge links, and gluon-gauge-link vertex. That is, we find that the topology in Fig. 2(d) is free of UV divergence if both external gluons are contracted by their respective momenta.

Similar to the discussion of the gluon-gauge-link vertex, the Ward identity helps reduce the superficial UV divergence of the topology in Fig. 2(d). Since the diagrams of the topology in Fig. 2(d) have only superficial logarithmic divergence, the additional reduction of the superficial UV divergence from the Ward identity makes the topology in Fig. 2(d) UV finite. Therefore, after the renormalization of topology in Fig. 2(c), the topology in Fig. 2(d) requires no additional renormalization. This is similar to the case of renormalizing gluon vertexes of QCD Lagrangian, where gauge invariance guarantees that the four-gluon vertex will be free of UV divergence once the three-gluon vertex is renormalized.

Summary.-We proved that UV divergences of all 36 pure quasigluon operators are localized in spacetime, and could be multiplicatively renormalized without mixing with either each other, or with quasiquark operators,

$$
\mathcal{O}_{g}^{\mu \nu \rho \sigma}(\xi)=e^{-C_{g}\left|\xi_{z}\right|} Z_{w g}^{-1} Z_{v g 1}^{-s / 2} Z_{v g 2}^{-(2-s) / 2} \mathcal{O}_{b g}^{\mu \nu \rho \sigma}(\xi),
$$

where $s$ is the number of $z$ components chosen for Lorentz indices $\{\mu, \nu, \rho, \sigma\}$, and $C_{g}, Z_{w g}, Z_{v g 1}$, and $Z_{v g 2}$ are renormalization constants. To achieve this result, it is crucial for the UV regulator to maintain gauge invariance. Our proof is obtained explicitly in continuum field theory using dimensional regularization.

In the case of lattice regularization, Lorentz symmetry is broken to the hypercubic symmetry while the gauge invariance is preserved. The gauge invariance and the reduced hypercubic symmetry should be sufficient for carrying through all arguments for the renormalization, and our final conclusion should not be changed.

Therefore, hadronic matrix elements of all operators defining quasiparton distributions could be examples of good "lattice cross sections," as defined in Refs. [9,10], which could be calculated in LQCD and factorized into normal PDFs, from which PDFs could be extracted by QCD global analysis of the data of these good "lattice cross sections" generated by the first principle LQCD calculations.

We thank Xu Feng, Luchang Jin, Anatoly Radyushkin, George Sterman, and Hong Zhang for useful discussions. This Letter is supported in part by the U.S. Department of Energy, Office of Science, Office of Nuclear Physics under Award No. DE-AC05-06OR23177, within the framework of the TMD Topical Collaboration.

Note added.-While our Letter is being finalized, a preprint by Zhang et al. [45] appeared, in which these authors reached the similar conclusion, although the approach is very different.

*lizhengyang@pku.edu.cn

yqma@pku.edu.cn

†jqu@ @lab.org

[1] J. C. Collins, D. E. Soper, and G. Sterman, Factorization of hard processes in QCD, Adv. Ser. Dir. High Energy Phys. 5, 1 (1988).

[2] H.-W. Lin et al., Parton distributions and lattice QCD calculations: A community white paper, Prog. Part. Nucl. Phys. 100, 107 (2018).

[3] X. Ji, Parton Physics on a Euclidean Lattice, Phys. Rev. Lett. 110, 262002 (2013).

[4] K.-F. Liu and S.-J. Dong, Origin of Difference Between Anti-d and Anti-u Partons in the Nucleon, Phys. Rev. Lett. 72, 1790 (1994).

[5] K.-F. Liu, Parton degrees of freedom from the path integral formalism, Phys. Rev. D 62, 074501 (2000).

[6] K.-F. Liu, Parton distribution function from the hadronic tensor on the lattice, Proc. Sci. LATTICE2015 (2016) 115.

[7] K. Orginos, A. Radyushkin, J. Karpie, and S. Zafeiropoulos, Lattice QCD exploration of parton pseudo-distribution functions, Phys. Rev. D 96, 094503 (2017).

[8] A. J. Chambers, R. Horsley, Y. Nakamura, H. Perlt, P. E. L. Rakow, G. Schierholz, A. Schiller, K. Somfleth, R. D. Young, and J.M. Zanotti, Nucleon Structure Functions from Operator Product Expansion on the Lattice, Phys. Rev. Lett. 118, 242001 (2017).

[9] Y.-Q. Ma and J.-W. Qiu, Extracting parton distribution functions from lattice QCD calculations, Phys. Rev. D 98, 074021 (2018).

[10] Y.-Q. Ma and J.-W. Qiu, Exploring Partonic Structure of Hadrons Using ab initio Lattice QCD Calculations, Phys. Rev. Lett. 120, 022003 (2018).

[11] T. Ishikawa, Y.-Q. Ma, J.-W. Qiu, and S. Yoshida, Practical quasi parton distribution functions, arXiv:1609.02018.

[12] J.-W. Chen, X. Ji, and J.-H. Zhang, Improved quasi parton distribution through Wilson line renormalization, Nucl. Phys. B915, 1 (2017).

[13] C. Monahan and K. Orginos, Quasi parton distributions and the gradient flow, J. High Energy Phys. 03 (2017) 116.

[14] R. A. Briceño, M. T. Hansen, and C. J. Monahan, Role of the Euclidean signature in lattice calculations of quasidistributions and other nonlocal matrix elements, Phys. Rev. D 96, 014502 (2017). 
[15] X. Xiong, T. Luu, and U.-G. Meißner, Quasi-parton distribution function in lattice perturbation theory, arXiv:1705 .00246 .

[16] M. Constantinou and H. Panagopoulos, Perturbative renormalization of quasi-parton distribution functions, Phys. Rev. D 96, 054506 (2017).

[17] C. Alexandrou, K. Cichy, M. Constantinou, K. Hadjiyiannakou, K. Jansen, H. Panagopoulos, and F. Steffens, A complete non-perturbative renormalization prescription for quasiPDFs, Nucl. Phys. B923, 394 (2017).

[18] J.-W. Chen, T. Ishikawa, L. Jin, H.-W. Lin, Y.-B. Yang, J.-H. Zhang, and Y. Zhao, Parton distribution function with nonperturbative renormalization from lattice QCD, Phys. Rev. D 97, 014505 (2018).

[19] X. Ji, J.-H. Zhang, and Y. Zhao, Renormalization in Large Momentum Effective Theory of Parton Physics, Phys. Rev. Lett. 120, 112001 (2018).

[20] J. Green, K. Jansen, and F. Steffens, Nonperturbative Renormalization of Nonlocal Quark Bilinears for QuasiPDFs on the Lattice Using an Auxiliary Field, Phys. Rev. Lett. 121, 022004 (2018).

[21] T. Ishikawa, Y.-Q. Ma, J.-W. Qiu, and S. Yoshida, Renormalizability of quasiparton distribution functions, Phys. Rev. D96, 094019 (2017).

[22] H.-W. Lin, J.-W. Chen, S. D. Cohen, and X. Ji, Flavor structure of the nucleon sea from lattice QCD, Phys. Rev. D 91, 054510 (2015).

[23] C. Alexandrou, K. Cichy, V. Drach, E. Garcia-Ramos, K. Hadjiyiannakou, K. Jansen, F. Steffens, and C. Wiese, Lattice calculation of parton distributions, Phys. Rev. D 92, 014502 (2015).

[24] J.-W. Chen, S. D. Cohen, X. Ji, H.-W. Lin, and J.-H. Zhang, Nucleon helicity and transversity parton distributions from lattice QCD, Nucl. Phys. B911, 246 (2016).

[25] C. Alexandrou, K. Cichy, M. Constantinou, K. Hadjiyiannakou, K. Jansen, F. Steffens, and C. Wiese, Updated lattice results for parton distributions, Phys. Rev. D 96, 014513 (2017).

[26] J.-H. Zhang, J.-W. Chen, X. Ji, L. Jin, and H.-W. Lin, Pion distribution amplitude from lattice QCD, Phys. Rev. D 95, 094514 (2017).

[27] C. Monahan, Smeared quasidistributions in perturbation theory, Phys. Rev. D 97, 054507 (2018).

[28] J.-W. Chen, T. Ishikawa, L. Jin, H.-W. Lin, A. Schäfer, Y.-B. Yang, J.-H. Zhang, and Y. Zhao, Gaussian-weighted Parton Quasi-distribution, arXiv:1711.07858.

[29] I. W. Stewart and Y. Zhao, Matching the quasiparton distribution in a momentum subtraction scheme, Phys. Rev. D 97, 054512 (2018).

[30] T. Izubuchi, X. Ji, L. Jin, I. W. Stewart, and Y. Zhao, Factorization theorem relating euclidean and light-cone parton distributions, Phys. Rev. D 98, 056004 (2018).
[31] C. Alexandrou, K. Cichy, M. Constantinou, K. Jansen, A. Scapellato, and F. Steffens, Reconstruction of LightCone Parton Distribution Functions from Lattice QCD Simulations at the Physical Point, Phys. Rev. Lett. 121, 112001 (2018).

[32] J.-W. Chen, L. Jin, H.-W. Lin, Y.-S. Liu, Y.-B. Yang, J.-H. Zhang, and Y. Zhao, Lattice calculation of parton distribution function from LaMET at physical pion mass with large nucleon momentum, arXiv:1803.04393.

[33] J.-W. Chen, L. Jin, H.-W. Lin, Y.-S. Liu, A. Schäfer, Y.-B. Yang, J.-H. Zhang, and Y. Zhao, First direct lattice-QCD calculation of the $x$-dependence of the pion parton distribution function, arXiv:1804.01483.

[34] Y.-S. Liu, J.-W. Chen, L. Jin, H.-W. Lin, Y.-B. Yang, J.-H. Zhang, and Y. Zhao, Unpolarized quark distribution from lattice QCD: A systematic analysis of renormalization and matching, arXiv:1807.06566.

[35] G. S. Bali, V. M. Braun, B. Gläßle, M. Göckeler, M. Gruber, F. Hutzler, P. Korcyl, A. Schäfer, P. Wein, and J.-H. Zhang, Pion distribution amplitude from Euclidean correlation functions: Exploring universality and higher twist effects, Phys. Rev. D 98, 094507 (2018).

[36] A. V. Radyushkin, Structure of parton quasi-distributions and their moments, Phys. Lett. B 788, 380 (2019).

[37] H.-W. Lin, J.-W. Chen, L. Jin, Y.-S. Liu, Y.-B. Yang, J.-H. Zhang, and Y. Zhao, Spin-Dependent Parton Distribution Function with Large Momentum at Physical Pion Mass, Phys. Rev. Lett. 121, 242003 (2018).

[38] J. Karpie, K. Orginos, and S. Zafeiropoulos, Moments of Ioffe time parton distribution functions from non-local matrix elements, J. High Energy Phys. 11 (2018) 178.

[39] W. Wang, S. Zhao, and R. Zhu, Gluon quasidistribution function at one loop, Eur. Phys. J. C 78, 147 (2018).

[40] W. Wang and S. Zhao, On the power divergence in quasi gluon distribution function, J. High Energy Phys. 05 (2018) 142.

[41] Z.-Y. Fan, Y.-B. Yang, A. Anthony, H.-W. Lin, and K.-F. Liu, Gluon Quasi-PDF From Lattice QCD, Phys. Rev. Lett. 121, 242001 (2018).

[42] A. M. Polyakov, Gauge fields as rings of glue, Nucl. Phys. B164, 171 (1980).

[43] V. Dotsenko and S. Vergeles, Renormalizability of phase factors in the nonabelian gauge theory, Nucl. Phys. B169, 527 (1980).

[44] J. C. Collins, Renormalization: An Introduction to Renormalization, the Renormalization Group, and the Operator-Product Expansion (Cambridge University Press, Cambridge, England, 1984), ISBN 0521311772.

[45] J.-H. Zhang, X. Ji, A. Schäfer, W. Wang, and S. Zhao, Renormalization of gluon quasi-PDF in large momentum effective theory, arXiv:1808.10824. 